\title{
Bloqueio do Nervo Isquiático por Abordagem Posterior Simplificada no Ponto Médio do Sulco Glúteo-Femoral: Estudo com Diferentes Volumes de Lidocaína a 1\% *
} Simplified Posterior Sciatic Nerve Block at Mid Gluteofemoral Sulcus:
Comparison of Different 1\% Lidocaine Volumes

\author{
Neuber Martins Fonseca, TSA ${ }^{1}$, Beatriz Lemos Mandim, TSA², Roberto Araújo Ruzi, TSA², Fabiana Rosa Tavares ${ }^{3}$.
}

\section{RESUMO}

Fonseca NM, Mandim BL, Ruzi RA, Tavares FR - Bloqueio do Nervo Isquiático por Abordagem Posterior Simplificada no Ponto Médio do Sulco Glúteo-Femoral: Estudo com Diferentes Volumes de Lidocaína a $1 \%$

JUSTIFICATIVA E OBJETIVOS: O bloqueio do nervo isquiático por via subglútea foi descrito com sucesso em estudo anterior, sendo mais uma opção entre as várias abordagens possíveis. $O$ nervo isquiático torna-se superficial na borda inferior do músculo glúteo máximo, permitindo seu acesso com fácil localização, pouco desconforto e baixo risco de punção acidental de grandes vasos. $O$ objetivo deste estudo foi avaliar o bloqueio do nervo isquiático por esta abordagem simplificada com diferentes volumes de lidocaína a 1\%.

MÉTODO: Foram estudados 40 pacientes com intervenções cirúrgicas na perna ou no pé distribuídos em dois grupos. Após monitorização, eles foram posicionados em decúbito ventral e realizado bloqueio no ponto médio do sulco glúteo-femoral, com auxílio de neuroestimulador e agulha de $5 \mathrm{~cm}$ eletricamente isolada, utilizando 300 mg (G1) ou 200 mg (G2) de lidocaína a 1\% sem adrenalina.

RESULTADOS: Obteve-se anestesia adequada em todos os casos com o volume e a concentração usados. $O$ tempo de execução do bloqueio foi de 8,6 \pm 5,7 $\mathrm{min}$ (G1) e 5,6 \pm 5,7 $\mathrm{min}$ (G2). A latência foi de 5,98 $\pm 1,4 \min (G 1)$ e 6,7 $\pm 2,9 \min (G 2)$. A duração sensitiva $e$ motora do bloqueio foi de $243 \pm 37$ min e $152 \pm 30$ min (G1) e $235 \pm$ $39 \min$ e $149 \pm 59$ min (G2), respectivamente. Não foram observadas diferenças estatísticas significativas entre os grupos estudados.

CONCLUSÕES: Essa abordagem é eficaz e de fácil execução, podendo a dose total de anestésico ser reduzida sem comprometimento da qualidade.

\footnotetext{
* Recebido (Received from) do Serviço de Anestesiologia (CET/SBA) da Faculdade de Medicina da Universidade Federal de Uberlândia (FM-UFU), Uberlândia, $M G$

1. Professor Doutor da Disciplina de Anestesiologia e Responsável pelo CET/ SBA da FM-UFU. Membro da Comissão de Normas Técnicas Segurança em Anestesiologia/SBA.

2. Co-Responsável pelo CET/SBA da FM-UFU.

3. Anestesiologista do Serviço de Anestesiologia (SEANE) do HCFM-UFU.
}

Apresentado (Submitted) em 19 de julho de 2005

Aceito (Accepted) para publicação 13 de fevereiro de 2006

Endereço para correspondência (Correspondence to)

Dr. Neuber Martins Fonseca

Rua Antônio Luiz Bastos, 300 Altamira

38411-116 Uberlândia, MG

E-mail:neuber@triang.com.br

(C) Sociedade Brasileira de Anestesiologia, 2006
Unitermos: ANATOMIA: nervo isquiático; ANESTESIA, Regional; ANESTÉSICOS, Local: lidocaína; TÉCNICAS ANESTÉSICAS, Regional: bloqueio do nervo isquiático.

\section{SUMMARY}

Fonseca NM, Mandim BL, Ruzi RA, Tavares FR - Simplified Posterior Sciatic Nerve Block at Mid Gluteofemoral Sulcus: Comparison of Different 1\% Lidocaine Volumes

BACKGROUND AND OBJECTIVES: Subgluteus sciatic nerve block has been successfully described in a previous study and is one more option among several possible approaches. The sciatic nerve becomes superficial at inferior gluteus maximus muscle where it is easily located and accessed with minor discomfort and low risk of accidental great vessels puncture. Our study aimed at evaluating this simplified sciatic nerve block approach with different $1 \%$ lidocaine volumes.

METHODS: Participated in this study 40 patients submitted to leg or foot procedures, who were distributed in two groups. Patients were placed in the prone position after monitoring and blockade was induced at mid gluteofemoral sulcus with the aid of neurostimulator and with beveled insulated $5 \mathrm{~cm}$ needle, with $300 \mathrm{mg}$ (G1) or 200 $m g$ (G2) of $1 \%$ plain lidocaine.

RESULTS: Used volumes and concentrations have promoted adequate anesthesia in all patients. Blockade time was $8.6 \pm 5.7 \mathrm{~min}$ (G1) and $5.6 \pm 5.7 \mathrm{~min}$ (G2). Onset time was $5.98 \pm 1.4 \mathrm{~min}$ (G1) and $6.7 \pm 2.9 \mathrm{~min}$ (G2). Sensory and motor block duration was 243 $\pm 37 \mathrm{~min}$ and $152 \pm 30 \mathrm{~min}$ (G1), and $235 \pm 39 \mathrm{~min}$ and $149 \pm 59$ min (G2), respectively. There were no statistically significant differences between groups.

CONCLUSIONS: This is an effective and easy approach and total anesthetic dose may be decreased without impairing quality.

Key Words: ANATOMY: sciatic nerve; ANESTHESIA, Regional; ANESTHETICS, Local: lidocaine; ANESTHETIC TECHNIQUES, Regional: sciatic nerve block

\section{INTRODUÇÃO}

$P$ ara que os bloqueios de nervos periféricos sejam procedimentos de rotina na prática clínica anestésica, algumas condições são necessárias, como serem práticas e simples de realizar, com fácil identificação dos pontos de referência, e não serem desconfortáveis para o paciente, pro- 
movendo anestesia adequada ao procedimento cirúrgico proposto. Apesar das várias propostas com diferentes vias de acesso para o bloqueio do nervo isquiático ${ }^{1-8}$, ele tem sido pouco realizado, por falta de adequado treinamento ou dificuldades técnicas ${ }^{9-11}$. Outro fator limitante para a aplicação prática desse bloqueio é a falta de padronização para via de acesso e a queixa de desconforto pelo paciente em muitas das técnicas propostas. Algumas limitações de aceitação relacionam-se com a dificuldade de identificação dos pontos de demarcação (sobretudo em pacientes obesos), enquanto outras, a dificuldades técnicas para realizar o bloqueio, por utilizar agulhas longas e que devem atravessar uma densa musculatura, como o glúteo, antes de atingir o ponto necessário ao bloqueio. Outras exigem injeções múltiplas, com o bloqueio dos nervos tibial e fibular na fossa poplítea.

Recentes estudos ${ }^{12,13}$ propõem simplificação da técnica do bloqueio do nervo isquiático por método de injeção única, agulha curta e eletricamente isolada, por abordagem posterior no ponto médio do sulco glúteo-femoral, identificando o nervo com auxílio de estimulador de nervo periférico. Esta considera a anatomia do nervo isquiático que emerge do plexo sacral, formado pelas raízes ventrais de $L_{4}$ a $S_{3}$, saindo da pelve através do forâmen isquiático maior, passando abaixo do músculo piriforme, descendo entre o trocânter maior do fêmur e a tuberosidade isquiática, e ao longo do dorso da coxa, anterior aos músculos bíceps femoral e semitendinoso, dirigindo-se para a fossa poplítea, onde se dividem em dois grandes ramos denominados nervos tibial e fibular comum. Essa disposição anatômica permite seu acesso por diferentes vias, do espaço parassacral à fossa poplítea.

A anestesia regional de membros inferiores tornar-se-á rotina, como pode ser observado atualmente com a anestesia do neuroeixo, quando as vantagens forem bem estabelecidas, sobretudo com redução dos efeitos adversos. Este fato foi observado por Fonseca e col. ${ }^{12}$, quando obtiveram bons resultados ao utilizar a técnica em pacientes com alterações cardiorrespiratórias sem aumento da morbimortalidade.

Por essa técnica, com abordagem simplificada no ponto médio do sulco glúteo-femoral, observa-se vantagem relacionada com a facilidade de identificação do ponto de referência, em contraposição à proposta de di Benedetto e col. ${ }^{8}$, que adotaram referências ósseas como o grande trocânter e a tuberosidade isquiática, desconsiderando o biotipo individual. Outra vantagem da técnica é o baixo risco de acidente vascular e impossibilidade de raquianestesia total ou peridural, como ocorre com o acesso proposto por Mansour e Bennetts ${ }^{14}$.

O grande diâmetro do nervo isquiático no nível do sulco glúteo-femoral poderia tornar a latência do bloqueio menos previsível que a de outros bloqueios periféricos. Essa possibilidade não se confirmou no estudo de Fonseca e col. ${ }^{12}$, que mostraram latência relativamente menor que outros estudos com outras vias de acesso e diferentes anestésicos 7,8,15-18. O espaço intermuscular no qual o nervo isquiático encontrase no sulco glúteo-femoral é pequeno e envolvido por redu- zida quantidade de tecido conjuntivo. Esta característica pode favorecer a impregnação do feixe nervoso pelo anestésico local. Assim, é possível pressupor que ele seja facilmente bloqueado com baixas doses e concentrações de anestésicos, diminuindo ainda mais a possibilidade de intoxicação anestésica, já descrita na realização do bloqueio do nervo isquiático ${ }^{19,20}$.

O objetivo deste estudo foi avaliar o bloqueio do nervo isquiático pela abordagem simplificada descrita por Fonseca e col. ${ }^{12}$, investigando o comportamento com diferentes volumes de lidocaína a $1 \%$.

\section{MÉTODO}

Após aprovação pelo Comitê de Ética em Pesquisa Clínica do Hospital de Clínicas da Faculdade de Medicina da Universidade Federal de Uberlândia e consentimento esclarecido dos pacientes, foram estudados de forma aleatória 40 pacientes, de ambos os sexos, estado físico ASA I a IV, com idade entre 21 e 82 anos, peso de 50 a $90 \mathrm{~kg}$, programados para serem anestesiados com a abordagem posterior do bloqueio do nervo isquiático e com intervenções cirúrgicas envolvendo a perna ou o pé. Foram distribuídos em dois grupos de igual número. O grupo 1 (G1) utilizou $30 \mathrm{~mL}$ de lidocaína a 1\% sem adrenalina (300 mg) e o grupo 2 (G2) $20 \mathrm{~mL}$ de lidocaína a $1 \%$ sem adrenalina $(200 \mathrm{mg})$. Foram excluídos do estudo os pacientes com contra-indicação à anestesia regional, portadores de doença psiquiátrica ou com história de abuso de drogas.

Antes do bloqueio anestésico, foi estabelecido acesso venoso com cateter $18 \mathrm{G}$ na veia do antebraço e infusão de solução de Ringer com lactato. Os pacientes foram monitorizados com eletrocardiograma contínuo na derivação $D_{\|}$ ou CM5, saturação periférica de oxigênio da oxiemoglobina $\left(\mathrm{SpO}_{2}\right)$, controle automático da pressão arterial pelo método oscilométrico durante a realização do bloqueio e no peri-operatório. O bloqueio foi realizado após sedação com 1 a 3 mg de midazolam por via venosa. Dose adicional de midazolam e fentanil na relação de $1 \mathrm{mg}$ e $50 \mu \mathrm{g}$, respectivamente, foi preparada para ser administrada ao paciente que se queixasse de desconforto ou necessitasse alívio complementar da ansiedade.

Para a realização do bloqueio todos os pacientes foram posicionados em decúbito ventral. Os critérios anatômicos considerados para realização do procedimento foram os propostos por Fonseca e col. ${ }^{12}$. A punção foi realizada no sulco glúteo-femoral. O ponto médio desse sulco foi adotado como local de referência para a introdução da agulha. Após antisepsia e anestesia da pele e do tecido subcutâneo com lidocaína a $1 \%$ sem vasoconstritor $(20 \mathrm{mg})$ introduziu-se agulha com bisel curto de $5 \mathrm{~cm}$ de comprimento e eletricamente isolada por teflon (Stimuplex A $50 \AA$, B.Braun) conectada a estimulador de nervo periférico (TOF- WATCH $\AA$ ), introduzida de forma perpendicular à pele. A agulha foi introduzida gradativamente com objetivo de atingir o nervo 
isquiático, localizado anteriormente ao músculo semitendinoso, passando pelo sulco delimitado pelos músculos semitendinoso, inserção do glúteo máximo e vasto lateral. O estímulo elétrico aplicado foi inicialmente ajustado com freqüência de $1 \mathrm{~Hz}$ e corrente de $1 \mathrm{~mA}$. A uma profundidade de 3 a $5 \mathrm{~cm}$ houve resposta de estímulo do componente tibial do nervo isquiático (inversão do pé e flexão plantar) ou do nervo fibular comum (dorsoflexão ou eversão do pé). Como a movimentação do pé representava resposta motora ao estímulo elétrico, a corrente foi reduzida a 0,5 mA. Caso o movimento desaparecesse, a posição da agulha era ajustada para manter resposta muscular com baixa corrente $(<0,5 \mathrm{~mA})$, e esse foi considerado o ponto ideal para a injeção do anestésico local programado para o grupo. O bloqueio foi realizado sem pesquisa de parestesia e a dose total programada administrada somente após dose-teste de $2 \mathrm{~mL}$ da solução anestésica, para afastar a possibilidade de injeção anestésica intraneural (dor aguda concomitante à injeção anestésica). A cada $5 \mathrm{~mL}$ injetados foi feita aspiração do êmbolo da seringa para confirmar que a agulha encontrava-se na região extravascular .

Todos os bloqueios foram realizados pelos autores. Nos procedimentos cirúrgicos em que havia indicação, foi realizado também bloqueio do nervo safeno (ramo sensitivo do nervo femoral). O nervo safeno foi abordado na altura do joelho e injetados $3 \mathrm{~mL}$ de lidocaína a 1\% (30 mg).

O paciente foi considerado como preparado para o procedimento cirúrgico quando apresentasse completa perda da sensação dolorosa à picada de agulha na distribuição dos nervos tibial e fibular, assegurada pela incapacidade de movimentação efetiva do tornozelo e pé.

Um observador independente, não envolvido com a realização do bloqueio, observou o tempo entre a introdução da agulha na pele e a resposta motora apropriada ao estímulo elétrico (tempo de realização do bloqueio), o tempo entre o final da administração do anestésico e o momento considerado apto para o procedimento cirúrgico (latência), o número de reposicionamentos da agulha necessários à resposta motora desejada, e a profundidade na qual a resposta ao estímulo elétrico ocorreu. A necessidade de analgesia ou sedação complementar foi anotada pelo mesmo observador independente.

Após o bloqueio, foi solicitado aos pacientes que avaliassem o desconforto durante o procedimento, usando uma escala de 3 pontos ( 1 = sem dor; 2 = dor moderada; $3=$ extremamente doloroso). Em todos os casos a intervenção cirúrgica foi conduzida sem necessidade complementar de anestesia. O bloqueio sensitivo foi verificado por aplicação de gelo no dorso (nervo fibular comum) e na região plantar do pé (nervo tibial), sendo considerado como completo quando o frio não era sentido. O bloqueio motor foi investigado solicitando-se ao paciente a realização de movimento de flexão dorsal e plantar do pé, e considerado como completo quando não ocorria movimento. A duração dos bloqueios motor e sensitivo foi definida como o período entre a injeção do anestésico e a recuperação das funções motora e sensitiva. A satisfação do paciente com a técnica anestésica foi avaliada 24 horas após o término do procedimento, por meio de uma escala de 2 pontos: 1 = satisfeito - "se fosse operar novamente aceitaria o mesmo tipo de anestesia". 2 = insatisfeito - "se fosse operar novamente gostaria que fosse realizado outro tipo de anestesia".

O estudo estatístico para avaliar a freqüência de sucesso de bloqueio, sexo, estado físico (ASA) entre os grupos foi feito pelo teste Exato de Fisher ou teste do Qui-quadrado. Idade, peso, duração da intervenção cirúrgica, intensidade do estímulo elétrico e a quantidade de reposicionamentos da agulha para realizar o bloqueio foi avaliada pelo teste $t$ de Student para análise de dados relativos a duas amostras que se apresentavam com escala intervalar ou de razão. $O$ teste $U$ de Mann-Whitney foi usado para comparar as latências sensitiva e motora entre os grupos. Em todos os testes, fixouse em $5 \%$ o nível para rejeição da hipótese de nulidade.

\section{RESULTADOS}

Nos 40 pacientes que participaram do estudo, o nervo isquiático foi identificado com resposta a estímulo mínimo de 0,5 mA. Não foram observadas diferenças de estímulo nos ramos tibial e fibular comum do nervo isquiático, assim como não foi relatado desconforto para a realização do bloqueio, não sendo necessária complementação sedativa para realização do procedimento anestésico. As características dos pacientes são mostradas na tabela I. Nela não é observada diferença significativa entre os grupos estudados.

O estudo do comportamento anestésico ao bloqueio do nervo isquiático é apresentado nas tabelas II, III e IV. Observou-

Tabela I - Descrição dos Dados Antropométricos dos Pacientes Estudados

\begin{tabular}{lccc}
\hline & $\begin{array}{c}\text { Grupo } 1 \\
(\mathrm{n}=20)\end{array}$ & $\begin{array}{c}\text { Grupo } 2 \\
(\mathrm{n}=20)\end{array}$ & $\mathrm{p}$ \\
\hline Idade (anos) * & $60,2 \pm 12,4$ & $58,9 \pm 17,8$ & $\mathrm{~ns}$ \\
Peso (kg) * & $57,1 \pm 5,8$ & $61,4 \pm 9,5$ & $\mathrm{~ns}$ \\
Altura (cm) * & $157,9 \pm 6,1$ & $162 \pm 7$ & $\mathrm{~ns}$ \\
Sexo & 12 & 6 & \\
Masculino & 8 & 14 & 0,2014 \\
Feminino & & & \\
Estado físico & 5 & 2 & 0,25 \\
ASA I & 4 & 6 & \\
ASA II & 11 & 10 & \\
ASA III & 0 & 2 & \\
ASA IV & & & \\
\hline
\end{tabular}

* Dados apresentados em Média \pm DP

ns - não significativa 
Tabela II - Descrição da Técnica de Identificação do Nervo Isquiático nos Pacientes Estudados

\begin{tabular}{lccc}
\hline & $\begin{array}{l}\text { Grupo 1 } \\
(\mathrm{n}=20)\end{array}$ & $\begin{array}{c}\text { Grupo 2 } \\
(\mathrm{n}=20)\end{array}$ & $\mathrm{p}$ \\
\hline $\begin{array}{l}\text { Identificação do } \\
\text { nervo isquiático (min) }\end{array}$ & 4 & 3 & 0,424889 \\
$\begin{array}{l}\text { Variação } \\
\begin{array}{l}\text { Reposicionamento } \\
\text { da agulha }\end{array}\end{array}$ & $2-18$ & $1-20$ & \\
$\begin{array}{l}\text { Variação } \\
\text { Profundidade da }\end{array}$ & $1-7$ & 5 & 0,002141 \\
agulha (cm) & 3,5 & 4 & 0,364848 \\
Variação & $2,5-5$ & $2,5-5$ & \\
\hline
\end{tabular}

Dados apresentados em mediana

Tabela III - Grau de Desconforto nos Pacientes Estudados durante a Realização do Bloqueio do Nervo Isquiático

\begin{tabular}{lcc}
\hline Graus & Grupo $1(\mathrm{n}=20)$ & Grupo $2(\mathrm{n}=20)$ \\
\hline 1 & 11 & 12 \\
2 & 9 & 7 \\
3 & 0 & 1 \\
\hline
\end{tabular}

1- sem dor; 2- dor moderada; 3- extremamente doloroso.

Tabela IV - Grau de Satisfação dos Pacientes Estudados quanto ao Bloqueio Realizado

\begin{tabular}{lcc}
\hline Graus & Grupo $1(n=20)$ & Grupo $2(n=20)$ \\
\hline 1 & 19 & 16 \\
2 & 1 & 4 \\
\hline
\end{tabular}

1- satisfeito com a técnica; 2- insatisfeito com a técnica

se diferença significativa na identificação do nervo isquiático no G2, não ocorrendo outro tipo de diferença entres os grupos estudados.

Não foi observada aspiração de sangue, parestesias, sinais clínicos de intoxicação anestésica durante ou após a realização da técnica para bloqueio do nervo isquiático. Não houve comprometimentos hemodinâmicos nem déficit sensitivo ou motor, após completa recuperação do bloqueio.

\section{DISCUSSÃo}

A investigação desenvolvida neste estudo visando diminuir a quantidade de anestésico, sem comprometer a qualidade do bloqueio do nervo isquiático pela via do subglúteo, mostrou resultados satisfatórios e promissores.

Significativa quantidade de pacientes avaliados eram portadores de vasculopatia periférica decorrente de complica- ções, sobretudo de diabete melito e, portanto, pacientes crônicos e de tratamento diversificado devido à multiplicidade de acometimento de órgãos. Apesar de o bloqueio do nervo isquiático ser pouco realizado na prática clínica, ele pode ser útil e a melhor opção em alguns pacientes, sobretudo naqueles com importante comprometimento do sistema cardiorrespiratório. A satisfação desses pacientes à realização do bloqueio foi gratificante pelo alívio do sofrimento, com recuperação rápida sem alteração dos parâmetros hemodinâmicos. Apesar deste trabalho não tê-la comparado com outras técnicas de bloqueio do nervo isquiático, essa observação corrobora com resultados de outros autores ${ }^{8,13,21}$.

O bloqueio de nervo periférico utilizando neuroestimulador permite a exata localização do fascículo nervoso sem necessidade de pesquisa de parestesia ${ }^{22}$. A injeção única associada à estimulação periférica relaciona-se com maior índice de sucesso do bloqueio. Praticamente não existem falhas do bloqueio quando a injeção da solução é feita após a indução de contrações musculares com corrente elétrica menor ou igual a $0,5 \mathrm{~mA}$, como já demonstrado em estudo anterior ${ }^{12}$ e confirmado neste, resultando na mesma qualidade de bloqueio mesmo com a redução do volume de anestésico local.

Alguns estudos afirmam que a técnica de múltiplas injeções é superior à da utilização do neuroestimulador ${ }^{22}$ e contraditório pelo maior risco teórico de trauma neurológico provocado pela agulha. A possibilidade de lesão neurológica é praticamente inexistente quando se utiliza material apropriado ao bloqueio, como agulhas eletricamente isoladas, de bisel curto e conectadas à estimulador de nervos periféricos regulado de forma correta ${ }^{22,23}$. Contudo, estudos demonstraram que alguns pacientes podem apresentar sintomas nas primeiras três semanas, mesmo após recuperação total do bloqueio, e que a grande maioria passa despercebida por resolução espontânea do quadro, dentro de até dez semanas, confundido com outros diagnósticos ${ }^{23}$, visto que em geral os mecanismos são multifatoriais ${ }^{24}$. Assim sendo, seria prematura qualquer afirmação de menor risco de complicações neurológicas pela técnica estudada, pelo não acompanhamento dos pacientes e por não ter havido comparação com outras técnicas. Tem sido referido como desconfortável e doloroso para o paciente o bloqueio do nervo isquiático por outras técnicas, sobretudo devido à passagem da espessa camada muscular que a agulha necessita atravessar para atingir regiões próximas ao nervo isquiático ${ }^{23}$. Os resultados deste estudo mostraram que a abordagem posterior pela via do sulco glúteo é efetiva para o bloqueio do nervo isquiático, sendo bem tolerada pelo paciente (Tabelas III e IV).

O grande diâmetro do nervo isquiático pode tornar a latência do bloqueio menos previsível que a de outros bloqueios periféricos. Apesar desse efeito farmacológico ser influenciado pelas propriedades químicas do anestésico utilizado, como a concentração e o volume ${ }^{11}$, outras interferem, destacandose o tipo de resposta motora obtida com o neuroestimulador ${ }^{22,23}$ ou a intensidade da corrente usada para estimular 0 
nervo ${ }^{22-26}$. Nesta investigação, a latência entre os dois grupos não mostrou diferença significativa, mas foi relativamente menor que em estudos prévios que utilizaram outros anestésicos por diferentes vias, como a ropivacaína, cuja latência média foi de 10 a 25 minutos ${ }^{13,14}$ ou levobupivacaína $8,13,22$. Como esses fatores foram mantidos constantes no presente estudo, o volume não influenciou os resultados.

$\mathrm{O}$ nervo isquiático é formado por fibras de $\mathrm{L}_{4}$ a $\mathrm{S}_{3}$. Dois troncos nervosos separados, os nervos tibial e fibular comum, são envolvidos por uma bainha epineural, distintos desde sua origem no plexo sacral. Esses dois troncos deixam a pelve através do forâmen isquiático maior, tornando-se superficiais no final do músculo glúteo máximo. Dirigem-se, então, para a fossa poplítea, onde se dividem em dois grandes ramos, denominados nervos tibial e fibular comum. Há demonstração anatômica da continuidade da bainha de tecido conjuntivo que envolve os troncos nervosos que compõem o nervo isquiático até sua divisão na fossa poplítea ${ }^{27}$. Essa particularidade anatômica é fundamental para justificar a redução do volume anestésico proposto por este estudo, quando não seria necessária a difusão cefálica ou caudal do anestésico para o efetivo bloqueio, pois o pequeno espaço intermuscular no qual o nervo isquiático encontra-se na região subglútea, associado à pequena quantidade de tecido conjuntivo, pode favorecer a impregnação do feixe nervoso pelo anestésico local. É provável que essa também seja a razão da curta latência e ausência de necessidade de suplementação analgésica no peri-operatório, diferente do que é referido por outros autores ${ }^{14}$.

Semelhante ao trabalho anterior ${ }^{12}$, não ocorreu anestesia da região correspondente ao nervo cutâneo posterior da coxa. A técnica apresenta vantagens substanciais, como facilidade de identificação do ponto de referência, baixo risco de lesão venosa e impossibilidade de complicações como raqui-total e peridural, descrita por Mansour e Bennetts ${ }^{14}$.

Conclui-se, por meio dos resultados deste estudo prospectivo, que o nervo isquiático pode ser bloqueado pela via posterior, com menor volume de anestésico local do que aquele inicialmente proposto.

\section{Simplified Posterior Sciatic Nerve Block at Mid Gluteofemoral Sulcus: Comparison of Different 1\% Lidocaine Volumes}

Neuber Martins Fonseca, TSA, M.D.; Beatriz Lemos Mandim, TSA, M.D.; Roberto Araújo Ruzi, TSA, M.D.; Fabiana Rosa Tavares, M.D.

\section{INTRODUCTION}

Some conditions are needed to routinely adopt peripheral nerve block in the anesthetic practice. It has to be technically simple, reference points have to be easily identified and approaches should not be uncomfortable for patients, promoting adequate anesthesia. Notwithstanding several proposals with different access pathways for sciatic nerve block ${ }^{1-8}$ it is seldom performed due to lack of adequate training or to technical difficulties ${ }^{9-11}$. Another limiting factor is the lack of access pathway standardization and patients' discomfort with some techniques. Some acceptance limitations are related to difficulties in identifying landmarks (especially in obese patients), while others are related to technical difficulties because blockade is induced with long needles which have to cross thick muscles, such as the gluteus, before reaching the blockade point. Others require multiple injections with tibial and fibular nerves block in the popliteal fossa.

Recent studies ${ }^{12,13}$ have proposed a simplified sciatic nerve block technique with single injection, short beveled insulated needle, by posterior approach at mid gluteofemoral sulcus, identifying the nerve with the aid of peripheral nerve stimulator. These techniques consider the anatomy of the sciatic nerve, which emerges from the sacral plexus, is formed by ventral roots $L_{4}$ to $S_{3}$, emerges from the pelvis through greater sciatic foramen, passes below the piriform muscle, goes down between the greater femoral trocanter and the sciatic tuberosity and along the dorsum of the thigh, anterior to femoral biceps and semitendinous muscles, going toward the popliteal fossa, where it is divided into two major branches called tibial and common fibular nerves. This anatomic position allows it to be accessed by different routes, from the parasacral space to the popliteal fossa.

Lower limbs regional anesthesia will become routine, as it is currently observed with neuraxial anesthesia, when its advantages are well established, especially with less adverse effects. This has been observed by Fonseca et al. ${ }^{12}$ who have obtained good results with the technique in patients with cardiopulmonary disorders without increasing morbiditymortality rates.

With this technique, with simplified approach at mid gluteofemoral sulcus, landmarks are easily identified, as opposed to the proposal of di Benedetto et al. ${ }^{8}$, who adopt bone references, such as greater trocanter and sciatic tuberosity, disregarding the individual biotype. Another advantage is the low risk of vascular accident and the impossibility of total spinal or epidural anesthesia, as it is the case with the access proposed by Mansour and Bennetts ${ }^{14}$.

The large sciatic nerve diameter at the level of the gluteofemoral sulcus could make blockade onset less predictable as compared to other peripheral blocks. This has not been confirmed by Fonseca et al. ${ }^{12}$ who have shown relatively shorter onset as compared to other studies with other access pathways and different anesthetic agents ${ }^{7,8,15-18}$.

The intermuscular space in which the sciatic nerve is located in the gluteofemoral sulcus is small and involved by a small amount of connective tissue. This may favor nervous bundle impregnation with local anesthetics. So, it is possible to suppose that it is easily blocked with low anesthetic doses and 
concentrations, further decreasing the possibility of anesthetic intoxication, already described during sciatic nerve block ${ }^{19,20}$. This study aimed at evaluating sciatic nerve block by the simplified technique described by Fonseca et al. ${ }^{12}$, investigating its behavior with different $1 \%$ lidocaine volumes.

\section{METHODS}

After the Clinical Research Ethical Committee, Hospital de Clínicas, Faculdade de Medicina, Universidade Federal, Uberlândia approval and their informed consent, participated in this study 40 patients of both genders, physical status ASA I to IV, aged 21 to 82 years, weighing 50 to $90 \mathrm{~kg}$, scheduled to be anesthetized with posterior sciatic nerve block and to be submitted to leg or foot procedures. Patients were randomly distributed in two equal groups. Group $1(\mathrm{G} 1)$ received $30 \mathrm{~mL}$ of $1 \%$ plain lidocaine (300 mg) and Group 2 (G2) received $20 \mathrm{~mL}$ of $1 \%$ plain lidocaine $(200 \mathrm{mg})$. Exclusion criteria were patients counterindicated for regional anesthesia, with psychiatric diseases or history of drug abuse.

Venous access was established before anesthetic block with $18 \mathrm{G}$ catheter in forearm vein and lactated Ringer's infusion. Patients were monitored with continuous ECG at $D_{\|}$or CM5 lead, peripheral hemoglobin oxygen saturation $\left(\mathrm{SpO}_{2}\right)$, and automatic blood pressure by the oscilometric method during blockade and in the intraoperative period. Blockade was induced after sedation with 1 to $3 \mathrm{mg}$ intravenous midazolam. Additional midazolam and fentanyl dose, in the ratio of $1 \mathrm{mg}$ and $50 \mu \mathrm{g}$, respectively, was prepared to administer to patients complaining of discomfort or needing additional anxiety relief.

Blockade was induced in the prone position in all patients. Anatomic criteria for the procedure were those proposed by Fonseca et al. ${ }^{12}$. Puncture was performed at the gluteofemoral sulcus. The mid point of this sulcus was adopted as landmark for needle introduction. After antisepsis and skin and subcutaneous tissue anesthesia with $1 \%$ plain lidocaine (20 mg), a short bevel, $5 \mathrm{~cm}$ insulated needle with Teflon (Stimuplex $A 50^{\circledR}$, B.Braun) connected to peripheral nerve stimulator (TOF-WATCH ${ }^{\circledR}$ ) was introduced perpendicular to skin. Needle was gradually introduced aiming at reaching the sciatic nerve located anteriorly to the semitendinous muscle, crossing the sulcus delimited by the semitendinous, gluteus maximus insertion and vastus lateralis muscles. Electrical stimulation was initially adjusted to $1 \mathrm{~Hz}$ frequency and $1 \mathrm{~mA}$ current. At the depth of 3 to $5 \mathrm{~cm}$, stimulation response of the tibial component of the sciatic nerve (foot inversion and plantar flexion) or of the common fibular nerve (dorsoflexion of foot eversion) was observed. Since foot movement represented motor response to electric stimulation, current was decreased to $0.5 \mathrm{~mA}$.

In the absence of movement, needle position was adjusted to maintain muscle response with low current $(<0.5 \mathrm{~mA})$, and this was considered the optimum point for local anesthetic injection. Blockade was performed without investigating pa- resthesia and total programmed dose was only administered after a test dose of $2 \mathrm{~mL}$ anesthetic solution to rule out the possibility of intraneural anesthetic injection (acute pain simultaneous to anesthetic injection). At every $5 \mathrm{~mL}$, syringe was aspired to confirm that the needle remained in the extravascular region.

All blockades were induced by the authors. When there was indication, the saphenous nerve was also blocked (sensory branch of the femoral nerve). Saphenous nerve was approached at the level of the knee and $3 \mathrm{~mL}$ of $1 \%$ lidocaine (30 $\mathrm{mg}$ ) were injected.

Patients were considered ready for the surgical procedure when they presented total loss of painful sensation at pinprick at the distribution of tibial and fibular nerves, confirmed by the inability to effective move ankle and foot.

An independent observer, not involved with the blockade, observed time between needle introduction and adequate motor response to electric stimulation (blockade time), time between end of anesthetic injection and the moment considered adequate for the procedure (onset), number of needle repositionings to obtain desired motor response, and depth in which there was electric stimulation response. The same independent observer recorded the need for additional analgesia or sedation.

After blockade, patients were asked to evaluate their discomfort during the procedure using a 3-point scale ( $1=$ no pain, 2 = moderate pain, 3 = extremely painful). All surgical procedures were performed with no need for anesthetic supplementation. Sensory block was confirmed by applying ice on foot dorsum (common fibular nerve) and plantar region (tibial nerve), being considered complete when cold was not felt. Motor block was confirmed by asking patients to move foot planta and dorsum, and was considered complete in the lack of movement. Motor and sensory block duration was defined as the period between anesthetic injection and motor and sensory function recovery. Patients' satisfaction with the technique was evaluated 24 hours post-procedure through a 2-point scale: 1 = satisfied - "if needing a new surgery, I would accept the same type of anesthesia"; 2 = unsatisfied - "if needing a new surgery, I would prefer a different type of anesthesia".

Fisher Exact or Chi-square tests were used to evaluate blockade success rate, gender and physical status (ASA) between groups. Age, weight, surgical procedure duration, electric stimulation intensity and number of needle repositioning for the blockade were evaluated by Student's $t$ test for analysis of data related to two samples presented with interval or ratio scale. Mann-Whitney $U$ test was used to compare sensory and motor onset between groups. Null hypothesis rejection level was $5 \%$ for all tests.

\section{RESULTS}

Sciatic nerve was identified in all 40 patients with minimum stimulation response of $0.5 \mathrm{~mA}$. There were no differences in stimulation of sciatic nerve, tibial and common fibular 
branches and there were no reports of discomfort with any need for sedative supplementation to perform the anesthetic procedure. Demographics data are shown in table I with no significant differences between groups.

Anesthetic behavior of sciatic nerve block is shown in tables II, III and IV. There has been significant difference in sciatic nerve identification in G2, without any other difference between groups.

Table I - Demographics Data

\begin{tabular}{lccc}
\hline & $\begin{array}{c}\text { Group 1 } \\
(\mathrm{n}=20)\end{array}$ & $\begin{array}{c}\text { Group 2 } \\
(\mathrm{n}=20)\end{array}$ & $\mathrm{p}$ \\
\hline Age (years) * & $60.2 \pm 12.4$ & $58.9 \pm 17.8$ & $\mathrm{~ns}$ \\
Weight $(\mathrm{kg}){ }^{*}$ & $57.1 \pm 5.8$ & $61.4 \pm 9.5$ & $\mathrm{~ns}$ \\
Height $(\mathrm{cm}){ }^{*}$ & $157.9 \pm 6,1$ & $162 \pm 7$ & $\mathrm{~ns}$ \\
Gender & & & \\
Male & 12 & 6 & 0.2014 \\
Female & 8 & 14 & \\
Physical status & & 2 & \\
ASA I & 5 & 6 & 0.25 \\
ASA II & 4 & 10 & \\
ASA III & 11 & 2 & \\
ASA IV & 0 & & \\
\hline
\end{tabular}

* Data expressed in Mean \pm SD

ns - non significant

Table II - Description of Sciatic Nerve Identification Technique

\begin{tabular}{lccc}
\hline & $\begin{array}{c}\text { Group 1 } \\
(\mathrm{n}=20)\end{array}$ & $\begin{array}{c}\text { Group 2 } \\
(\mathrm{n}=20)\end{array}$ & $\mathrm{p}$ \\
\hline $\begin{array}{l}\text { Sciatic nerve } \\
\text { identification (min) }\end{array}$ & 4 & 3 & 0.424889 \\
Variation & $2-18$ & $1-20$ & \\
Needle repositioning & 2 & 5 & 0.002141 \\
Variation & $1-7$ & $1-10$ & \\
Needle depth (cm) & 3,5 & 4 & 0.364848 \\
Variation & $2.5-5$ & $2.5-5$ & \\
\hline
\end{tabular}

Data presented in median

Table III - Rating of Patients' Discomfort during Sciatic Nerve Block

\begin{tabular}{lcc}
\hline Rating & Group 1 $(\mathrm{n}=20)$ & Group 2 $(\mathrm{n}=20)$ \\
\hline 1 & 11 & 12 \\
2 & 9 & 7 \\
3 & 0 & 1 \\
\hline
\end{tabular}

1- no pain; 2- moderate pain; 3- extremely paintful
Table IV - Rating of Patients' Satisfaction with Blockade

\begin{tabular}{lcc}
\hline Rating & Group 1 $(\mathrm{n}=20)$ & Group 2 $(\mathrm{n}=20)$ \\
\hline 1 & 19 & 16 \\
2 & 1 & 4 \\
\hline 1- satisfied; 2- unsatisfied &
\end{tabular}

No blood aspiration, paresthesia or clinical signs of anesthetic intoxication during or after sciatic nerve block were observed. There were no hemodynamic changes. There has been no sensory or motor deficit after total blockade recovery.

\section{DISCUSSION}

The investigation developed in this study to decrease anesthetic dose without impairing sciatic block quality via subgluteus has shown satisfactory and promising results.

A large number of patients presented peripheral vasculopathy due to pathological complications, especially diabetes mellitus, thus being chronically ill patients with diversified treatment modalities with the involvement of several organs. Although sciatic nerve block is seldom performed in the clinical practice, it may be useful and the best option for some patients, especially those with major cardiopulmonary disorders. Patients' satisfaction with blockade was gratifying due to suffering relief with fast recovery without hemodynamic changes. Although it has not compared this technique to other sciatic nerve block techniques, this observation confirms results of other authors ${ }^{8,13,21}$.

Peripheral nerve block with neurostimulator allows the exact location of the nervous fascicle with no need for investigating paresthesia ${ }^{22}$. Single injection associated to peripheral stimulation is associated to higher blockade success rate. There are virtually no blockade failures when solution is injected after the induction of muscle contractions with electric current equal to or below $0.5 \mathrm{~mA}$, as shown in a previous study ${ }^{12}$ and confirmed by this study, resulting in the same blockade quality even with decreased local anesthetic volume.

Some studies state that multiple injections technique is better as compared to neurostimulator ${ }^{22}$, which is contradictory due to higher theoretical risk of needle-induced neurological trauma. There is virtually no risk of neurological injury when adequate material is used, with short beveled insulated needles connected to adequately adjusted peripheral nerve stimulator ${ }^{22,23}$. However, studies have shown that some patients may have symptoms in the first three weeks, even after total blockade recovery, and that the vast majority goes unnoticed due to spontaneous resolution within up to 10 weeks, being mistaken with other diagnoses ${ }^{23}$ since mechanisms are in general multifactoral ${ }^{24}$. So, any statement of lower risk of neurological complications by the studied technique would be premature because patients were not followed-up and there were no comparisons with other techniques. 
Sciatic nerve block by other techniques has been referred as uncomfortable especially due to the thick muscle layer the needle has to cross to reach regions close to the sciatic nerve ${ }^{23}$. The study results have shown that the posterior approach via gluteus sulcus is effective for sciatic nerve block, being well tolerated by patients (Tables III and IV).

The large sciatic nerve diameter may make blockade onset less predictable as compared to other peripheral blocks. Although this pharmacological effect is influenced by chemical anesthetic properties, such as concentration and volume ${ }^{11}$, others may also interfere, such as type of motor response obtained with the neurostimulator ${ }^{22,23}$ or the current intensity used to stimulate the nerve ${ }^{22-26}$.

In this study, there has been no significant difference in onset between groups, but it was relatively faster as compared to previous studies using other anesthetic agents and different routes, such as ropivacaine, the mean onset of which was 10 to 25 minutes ${ }^{13,14}$, or levobupivacaine ${ }^{8,13,22}$. Since these factors were maintained constant in our study, volume has not influenced results.

The sciatic nerve is made up of $L_{4}$ to $S_{3}$ fibers. Two separate nervous branches, tibial and common fibular nerves, are involved by an epineural sheath and are distinct since their origin in the sacral plexus. These two branches leave the pelvis through the greater sciatic foramen and become superficial at the end of the gluteus maximum muscle. Then they go to the popliteal fossa where they divide into two major branches called tibial and common fibular nerve. There is anatomic demonstration of the continuity of the connective tissue sheath involving nervous branches making up the sciatic nerve until its division in the popliteal fossa ${ }^{27}$.

This anatomic uniqueness is fundamental to justify the lower anesthetic volume proposed in our study, when no cephalad or caudal anesthetic spread would be needed for an effective blockade, because the small intermuscular space in which the sciatic nerve is inserted in the subgluteus region, associated to small amount of connective tissue, may favor nervous bundle impregnation by local anesthetics. This may also be the possible reason for the fast onset and the lack of perioperative analgesic supplementation, differently from what has been reported by other authors ${ }^{14}$

Similar to previous study ${ }^{12}$, there was no anesthesia in the region corresponding to the posterior cutaneous nerve of thigh. The technique has substantial advantages, such as easy landmark, low risk of venous injury and impossibility of complications such as total spinal or epidural anesthesia described in Mansour and Bennetts ${ }^{14}$.

The conclusion of this prospective study is that the sciatic nerve may be blocked by the posterior pathway, with lower local anesthetic volume than that initially proposed.

\section{REFERÊNCIAS - REFERENCES}

01. Ichiyanagi $\mathrm{K}$ - Sciatic nerve block: lateral approach with patient supine. Anesthesiology, 1959;20:601-604.
02. Labat G - Its Technique and Clinical Applications: Regional Anaesthesia, $2^{\text {nd }}$ Ed, Philadelphia, WB Saunders, 1924;45-55.

03. Raj PP, Parks RI, Watson TD et al - A new single-position supine approach to sciatic-femoral nerve block. Anesth Analg, 1975;54:489-493.

04. Beck GP - Anterior approach to sciatic nerve block. Anesthesiology, 1963;24:222-224.

05. Rorie DK, Byer DE, Nelson DO et al - Assessment of block of the sciatic nerve in the popliteal fossa. Anesth Analg, 1980; 59:371-376.

06. McLeod DH, Wong DH, Claridge RJ et al - Lateral popliteal sciatic nerve block compared with subcutaneous infiltration for analgesia following foot surgery. Can J Anaesth, 1994;41:673-676.

07 . Chelly JE, Delaunay $L-A$ new anterior approach to the sciatic nerve block. Anesthesiology, 1999;91:1655-1660.

08. di Benedetto $P$, Bertini $L$, Casati $A$ et al $-A$ new posterior approach to the sciatic nerve block: a prospective, randomized comparison with the classic posterior approach. Anesth Analg, 2001; 93:1040-1044.

09. Hadzic A, Vloka JD, Kuroda MM et al - The practice of peripheral nerve blocks in the United States: a national survey. Reg Anesth Pain Med, 1998;23:241-246.

10. Bouaziz H, Mercier FJ, Narchi P et al - Survey of regional anesthetic practice among French residents at time of certification. Reg Anesth, 1997;22:218-222.

11. Smith MP, Sprung J, Zura A et al - A survey of exposure to regional anesthesia techniques in American anesthesia residency training programs. Reg Anesth Pain Med, 1999;24:11-16.

12. Fonseca NM, Ferreira FX, Ruzi RA et al - Abordagem simplificada do nervo isquiático por via posterior, no ponto médio do sulco glúteo-femoral, com uso de neuroestimulador. Rev Bras Anestesiol, 2002;52:764-773.

13. Sukhani R, Candido KD, Doty R Jr et al - Infragluteal-parabiceps sciatic nerve block: an evaluation of a novel approach using a single-injection technique. Anesth Analg, 2003;96:868-873.

14. Mansour NY, Bennetts FE - An observational study of combined continuous lumbar plexus and single-shot sciatic nerve blocks for post-knee surgery analgesia. Reg Anesth, 1996; 21:287-291.

15. McClellan KJ, Faulds D - Ropivacaine: an update of its use in regional anaesthesia. Drugs, 2000;60:1065-1093.

16. Casati A, Fanelli G, Borghi B et al - Ropivacaine or $2 \%$ mepivacaine for lower limb peripheral nerve blocks. Study Group on Orthopedic Anesthesia of the Italian Society of Anesthesia, Analgesia, and Intensive Care. Anesthesiology, 1999;90:1047-1052.

17. Casati A, Magistris L, Fanelli G et al - Small-dose clonidine prolongs postoperative analgesia after sciatic-femoral nerve block with $0.75 \%$ ropivacaine for foot surgery. Anesth Analg, 2000;91:388-392.

18. Coventry DM, Todd JG - Alkalinisation of bupivacaine for sciatic nerve blockade. Anaesthesia, 1989;44:467-470.

19. Petitjeans F, Mion G, Puidupin M et al - Tachycardia and convulsions induced by accidental intravascular ropivacaine injection during sciatic block. Acta Anaesthesiol Scand, 2002; 46:616-617.

20. Ruetsch YA, Fattinger KE, Borgeat A - Ropivacaine-induced convulsions and severe cardiac dysrhythmia after sciatic block. Anesthesiology, 1999;90:1784-1786.

21. Taboada M, Alvarez $\mathrm{J}$, Cortes $\mathrm{J}$ et al - The effects of three different approaches on the onset time of sciatic nerve blocks with $0.75 \%$ ropivacaine. Anesth Analg, 2004;98:242-247.

22. Fanelli G, Casati A, Garancini $P$ et al - Nerve stimulator and multiple injection technique for upper and lower limb blockade: failure rate, patient acceptance, and neurologic complications. Study Group on Regional Anesthesia. Anesth Analg, 1999; 88:847-852. 
23. Smith BE, Allison A - Use of a low-power nerve stimulator during sciatic nerve block. Anaesthesia, 1987;42:296-298.

24. Auroy $Y$, Narchi $P$, Messiah $A$ et al - Serious complications related to regional anesthesia: results of a prospective survey in France. Anesthesiology, 1997;87:479-486.

25. Liguori GA - Complications of regional anesthesia: nerve injury and peripheral neural blockade. J Neurosurg Anesthesiol, 2004;16:84-86.

26. Shah S, Hadzic A, Vloka JD et al - Neurologic complications after anterior sciatic nerve block. Anesth Analg, 2005;100:15151517.

27. Vloka JD, Hadzic A, Lesser JB et al - A common epineural sheath for the nerves in the popliteal fossa and its possible implications for sciatic nerve block. Anesth Analg, 1997;84:387-390.

\section{RESUMEN}

Fonseca NM, Mandim BL, Ruzi RA, Tavares FR - Bloqueo del Nervio Isquiático por Abordaje Posterior Simplificado en el Punto Medio del Surco Glúteo-Femoral: Estudio con Diferentes Volúmenes de Lidocaína a $1 \%$.

JUSTIFICATIVA Y OBJETIVOS: El bloqueo del nervio isquiático por vía subglútea fue descrito con éxito en estudio anterior, siendo una opción más entre los varios abordajes posibles. El nervio isquiático se hace superficial en el borde inferior del músculo glúteo máximo, permitiendo su acceso con fácil ubicación, poca incomodidad y bajo riesgo de punción accidental de grandes vasos. El objetivo de este estudio fue el de evaluar el bloqueo del nervio isquiático por este abordaje simplificado con diferentes volúmenes de lidocaína a $1 \%$.

MÉTODO: Se estudiaron 40 pacientes con intervenciones quirúrgicas en la pierna o en el pie, distribuidos en dos grupos. Después de la monitorización, fueron colocados en decúbito ventral y realizado el bloqueo en el punto medio del surco glúteo-femoral, con auxilio de neuroestimulador y aguja de $5 \mathrm{~cm}$, eléctricamente aislada, utilizando 300 mg (G1) o 200 mg (G2) de lidocaína a 1\% sin adrenalina.

RESULTADOS: Se obtuvo anestesia adecuada en todos los casos con el volumen y la concentración utilizados. El tiempo de ejecución del bloqueo fue de 8,6 \pm 5,7 $\min$ (G1) y 5,6 5,7 min (G2). La latencia fue de 5,98 $\pm 1,4 \mathrm{~min}$ (G1) y 6,7 $\pm 2,9 \mathrm{~min}$ (G2). La duración sensitiva y motora del bloqueo fue de $243 \pm 37$ min y 152 \pm 30 min (G1) y $235 \pm 39$ min y $149 \pm 59$ min (G2), respectivamente. No se observaron diferencias estadísticas significativas entre los grupos estudiados.

CONCLUSIONES: Ese abordaje es eficaz y de fácil ejecución, pudiendo la dosis total de anestésico ser reducida sin el comprometimiento de la calidad. 